\title{
Rhinovirus species/genotypes and interferon- $\lambda$ : subtypes, receptor and polymorphisms - missing pieces of the puzzle of childhood asthma?
}

To the Editor:

We agree with BERGAUER et al. [1]: in order to understand and manage asthma it is necessary to characterise better the virological and immunological factors associated with exacerbation events. In their article, those authors indicated that human rhinovirus (HRV) infection may regulate type I and III interferon (IFN) expression/production in paediatric asthma, both at baseline and during disease exacerbations. Although these findings are interesting and meaningful, we are concerned about some shortcomings in their experimental design and discussion that might lead to misleading conclusions.

First, the importance of HRV species (A, B and C) as a significant factor in causing asthma exacerbations and, indirectly, in type I and III IFN activation in children was not considered at all by the authors, while it is known that HRV-C is associated with wheezing and asthma hospitalisation more often than the other species [2,3]. Furthermore, as expected, genotyping of HRV in 140 hospitalised children recently allowed us to establish that: the frequencies of HRV-A or HRV-C may change depending on the specific respiratory disease, HRV-C being the more common in asthmatic bronchitis (figure 1a); and a large number of genotypes of either HRV-A or -C may be associated with paediatric hospitalisation (figure 1a).

Second, it is not clear why the authors sampled blood to measure type I and III IFN response. It is known that IFN- $\lambda$ may primarily act as protection in different regions at the mucosal level, such as the lung, skin or digestive tract, due the fact that the IFN- $\lambda$ receptor is preferentially expressed on epithelial cells [4]. The ability of these cells to release IFN- $\lambda$ after viral infections is also indicated by the fact that IFN- $\lambda$ expression seems to be favoured when activated MAVS (mitochondrial antiviral signalling) protein localises to peroxisomes, which are mainly abundant in epithelial cells [5]. These considerations, together with the knowledge that historically, the proper way to evaluate in vivo IFN expression is to measure the IFN-stimulated gene expression in biological fluids instead of the IFN protein or its biological activity itself (due to the low half-life of circulating IFN proteins and their extremely low levels (generally on the order of femtograms)), might, at least in part, simply explain the reasons why the authors found many "not determined" samples among sera from children suffering from asthma. Moreover, since type III IFN comprises four different subtypes, IFN- $\lambda 1-4$, the latter of which is tightly regulated by a dinucleotide polymorphism [6], the reason behind the choice of the authors to measure only IFN- $\lambda 1$ is not clear. Thus, in our opinion, a definite conclusion cannot be drawn on this issue.

Third, due to the specific and restricted expression of type III receptors, we retain that the evaluation of its expression could be fundamental in order to characterise the role of IFN- $\lambda$ in any of the analysed regions. Interestingly, levels of IFN- $\lambda$ receptor 1 (also known as interleukin $28 \mathrm{~B}$ receptor 1 ) was higher in nasopharyngeal washings of infants hospitalised for HRV bronchiolitis compared to those with RSV bronchiolitis (figure 1b). This finding, together with our previous observations indicating the presence of impaired airway IFN- $\lambda 1-3$, pattern recognition receptors and IFN-stimulated gene expression in HRV infection [7-9], make more evident the complexity of the analysed phenomenon, and the need to be very careful and cautious in the interpretation of the data.

@ERSpublications

Rhinovirus and interferon- $\lambda$ : missing pieces of the puzzle of childhood asthma http://ow.ly/vBL9308zN20

Cite this article as: Scagnolari C, Midulla F, Nenna R, et al. Rhinovirus species/genotypes and interferon- $\lambda$ : subtypes, receptor and polymorphisms - missing pieces of the puzzle of childhood asthma? Eur Respir J 2017; 49: 1700060 [https://doi.org/10.1183/13993003.00060-2017]. 

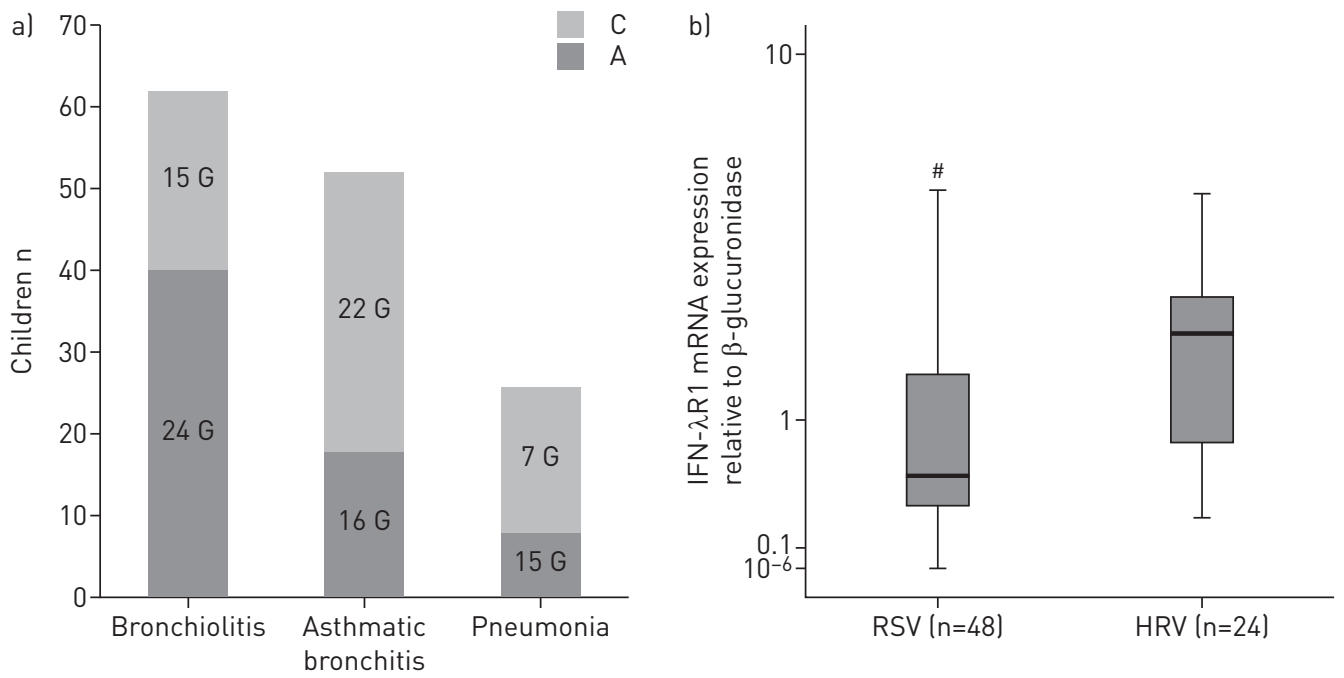

FIGURE 1 Molecular evaluation of human rhinovirus (HRV) species and interferon $-\lambda$ receptor 1 (IFN- $\lambda$ R1) expression in children suffering from respiratory diseases. a) Histograms show the distribution of HRV-A or HRV-C species and the related number of genotypes $(G)$ in children hospitalised for bronchiolitis $(n=62$, median (range) age 2 (0.3-17) months, males/females 31/31), asthmatic bronchitis ( $n=52$, age 9 (0.1617) months, males/females $35 / 17)$ or pneumonia ( $n=26$, age 24 (0.5-177) months, males/females 10/16). HRV was genotyped by sequencing the 390 bases of central portion of the $5^{\prime}$ untranslated region. The frequency of HRV-A or HRV-C varied according to the specific respiratory disease considered ( $p=0.004$ using Chi-squared test). b) Gene expression of IFN- $\lambda \mathrm{R} 1$ in nasopharyngeal washings collected in the first $48 \mathrm{~h}$ after admission to the hospital from infants suffering from respiratory syncytial virus (RSV) ( $n=48$, age 2 (1-12) months; males/ females 26/22) or HRV ( $n=24$, age 2 (1-12) months, males/females 11/13) associated bronchiolitis. The mRNA copy content of IFN- $\lambda$ R1 was measured by a real-time $5^{\prime}$ exonuclease RT-PCR assay using the Light Cycler 480 sequence detector (Roche Diagnostics SpA, Monza, Italy). The co-amplification of the $\beta$-glucuronidase gene was used to normalise the amount of total RNA present using the threshold cycle relative quantification method. Levels of IFN- $\lambda$ R1 were higher in HRV than in RSV infection. ${ }^{\#}$ : $p=0.017$ using Mann-Whitney test.

In conclusion, we believe that understanding the host-HRV interactions that determine the severity of respiratory illnesses and long-term sequelae would be of great help in identifying at-risk patients, and in designing new and more effective therapeutic and preventive strategies. In this regard, BERGAUER et al. [1] should undoubtedly be merited with having addressed such an issue, and having increased our understanding of the importance of IFN $-\alpha$ and $-\lambda$ production/expression in asthmatic children [10]; however, we maintain that the complex puzzle of childhood asthma regarding HRV and type I and III IFN response is still far from being solved.

Carolina Scagnolari $\odot^{1}$, Fabio Midulla ${ }^{2}$, Raffaella Nenna ${ }^{2}$, Paola Papoff ${ }^{2}$, Guido Antonelli ${ }^{1}$ and Alessandra Pierangeli ${ }^{1}$ ${ }^{1}$ Dept of Molecular Medicine, Laboratory of Virology affiliated to Istituto Pasteur Italia - Fondazione Cenci Bolognetti, Sapienza University, Rome, Italy. ${ }^{2}$ Dept of Pediatrics and Infantile Neuropsychiatry, Sapienza University Rome, Rome, Italy.

Correspondence: Carolina Scagnolari, Dept of Molecular Medicine, Laboratory of Virology affiliated to Istituto Pasteur Italia - Fondazione Cenci Bolognetti, Sapienza University, Porta Tiburtina 28, Rome, Italy.

E-mail: carolina.scagnolari@uniroma1.it

Received: Jan 112017 | Accepted: Jan 122017

Support statement: This work was supported by a grant to G. Antonelli and F. Midulla from Sapienza University of Rome (Ricerche universitarie 2015/16). Funding information for this article has been deposited with the Open Funder Registry.

Conflict of interest: None declared.

\section{References}

1 Bergauer A, Sopel N, Kroß B, et al. IFN- $\alpha /$ IFN- $\lambda$ responses to respiratory viruses in paediatric asthma. Eur Respir J 2017; 49: 1600969.

2 Gern JE. The ABCs of rhinoviruses, wheezing, and asthma. J Virol 2010; 84: 7418-7426.

3 Stone CA Jr, Miller EK. Understanding the association of human rhinovirus with asthma. Clin Vaccine Immunol 2015; 23: 6-10.

4 Hermant $\mathrm{P}$, Michiels T. Interferon $-\lambda$ in the context of viral infections: production, response and therapeutic implications. J Innate Immun 2014; 6: 563-574.

5 Odendall C, Dixit E, Stavru F, et al. Diverse intracellular pathogens activate type III interferon expression from peroxisomes. Nat Immunol 2014; 15: 717-726. 
6 Prokunina-Olsson L, Muchmore B, Tang W, et al. A variant upstream of IFNL3 (IL28B) creating a new interferon gene IFNL4 is associated with impaired clearance of hepatitis C virus. Nat Genet 2013; 45: 164-171.

7 Selvaggi C, Pierangeli A, Fabiani M, et al. Interferon lambda 1-3 expression in infants hospitalized for RSV or HRV associated bronchiolitis. J Infect 2014; 68: 467-477.

8 Scagnolari C, Midulla F, Pierangeli A, et al. Gene expression of nucleic acid-sensing pattern recognition receptors in children hospitalized for respiratory syncytial virus-associated acute bronchiolitis. Clin Vaccine Immunol 2009; 16: 816-823.

9 Scagnolari C, Midulla F, Trombetti S, et al. Upregulation of interferon-induced genes in infants with virus-associated acute bronchiolitis. Exp Biol Med (Maywood) 2007; 232: 1355-1359.

10 Johnston SL. Innate immunity in the pathogenesis of virus-induced asthma exacerbations. Proc Am Thorac Soc 2007; 4: 267-270. 